\title{
Intragastric rupture of splenic artery pseudoaneurysm after sleeve gastrectomy: A case-report
}

\author{
Ricci D ${ }^{1}$, Dell'Aversano Orabona $\mathrm{G}^{2 *}$, Emili $\mathbf{I}^{1}$, Serpi $\mathrm{F}^{1}$, Sgrazzutti $\mathrm{C}^{1}$ and Vanzulli $\mathrm{A}^{1}$ \\ ${ }^{1}$ Department of Radiology, University of Milan, Milan, Italy \\ ${ }^{2}$ Department of Advanced Biomedical Sciences, "Federico II" University of Naples, Via S. Pansini, Naples, Italy
}

\begin{abstract}
Splenic artery aneurysms are the third most common intra-abdominal aneurysm. Splenic artery pseudoaneurism are extremely rare. Despite the histologic differences in their wall composition, clinical findings and incidence, treatment should be always undertaken in case of increased risk of rupture. We present a patient with massive upper GI bleeding treated with laparotomy because of hemodynamic compromise. He had laparotomic Sleeve gastrectomy for obesity 2 years before the hemorrhagic event. The cause of the bleed was found to be a pseudoaneurysm of the splenic artery.
\end{abstract}

\section{Introduction}

Splenic artery pseudoaneurysms are a rare type of pseudoaneurysm arising from any portion of the splenic artery and its branches [1].

They are distinct entity and are different from true aneurysms of the splenic artery. In true aneurysm, wall is composed of intima, media, and adventitia; on the other hand, pseudoaneurysm wall contains only intima, media and fibrous tissue and they are even more rare than true aneurysms [2].

Their size can vary and if larger than $5 \mathrm{~cm}$ they are called giant splenic artery pseudoaneurysms [3].

While splenic artery aneurysms (SAAs) are the third most common intra-abdominal aneurysm after those of the aortic and iliac artery [4] and atherosclerotic changes are observed in up to $99 \%$ of the SAAs histologically examined [5], in splenic pseudoaneurism atherosclerosis is rarely the primary causative factor [6]. The causes of splenic artery pseudoaneurysm include pancreatitis, trauma, iatrogenic and previous surgery, and, rarely, peptic ulcer disease and often presentation is with symptoms [1].

Moreover, splenic artery pseudoaneurism after an operative procedure is extremely rare and is hardly suspected [7].

We present a patient with massive upper GI bleeding treated with laparotomy because of hemodynamic compromise. He had Sleeve gastrectomy for obesity 2 years before the haemorrhagic event. The cause of the intra-gastric bleed was found to be a pseudoaneurysm of the splenic artery.

\section{Case report}

A 49-year-old woman was admitted in the emergency room (ER) after presenting with epigastric pain. Her past history was significant for an open Sleeve gastrectomy 2 years ago. Her weight reduced of $56 \mathrm{~kg}$ after the obesity surgery. On this admission, haemoglobin was found to have dropped from 12.3 to $8.7 \mathrm{~g} / \mathrm{dl}$ in a few hours. After a non conclusive abdominal radiography, emergency CT examination was performed before and after iodinated contrast media intravenous injection, with arterial and venous phases of study.

CT Imaging findings suggested the presence of splenic artery aneurysmatic dilatation, which can't be separeted from the gastric wall, close to surgical clips of the previous Sleeve gastrectomy (Figure 1). A mediastinal hematoma constricted the esophagus (Figure 2). Fat stranding of the retroperitoneal space, hemoperitoneum (Figure 3) and ischemic injuries of the spleen were present (Figure 4).

The source of bleeding could not be immediately identified, but our first diagnostic hypothesis was the rupture of voluminous splenic pseudo-aneurysm involving the gastric wall in correspondence of surgical clips line. The presence of persistent hemodynamic instability of our patient led to emergency laparotomy. In the operating room, aspiration of blood clots $(2800 \mathrm{cc})$ was performed and a fistula between the splenic artery pseudoaneurysm $(2 \mathrm{~cm})$ and the lateral gastric wall was found.

Isolation of splenic artery from gastric wall and temporary hemostasis were obtained and splenectomy due to ischemic injury was performed.

The patient had a prolonged recovery but left the hospital in good condition.

The history of previous surgery and the successive histologic examination confirmed the presence of splenic pseudoaneurysm.

\section{Discussion}

Pseudoaneurysms are entity similar to SAAs, but more common in men and generally result from the destruction of the whole arterial wall by chronic pancreatitis, septic emboli (mycotic aneurysm), or

${ }^{*}$ Correspondence to: Dell'Aversano Orabona G, Department of Advanced Biomedical Sciences, "Federico II" University of Naples, Via S. Pansini, Naples, Italy, E-mail: giuseppinadellaversanoorabona@gmail.com

Received: June 03, 2019; Accepted: June 11, 2019; Published: June 14, 2019 

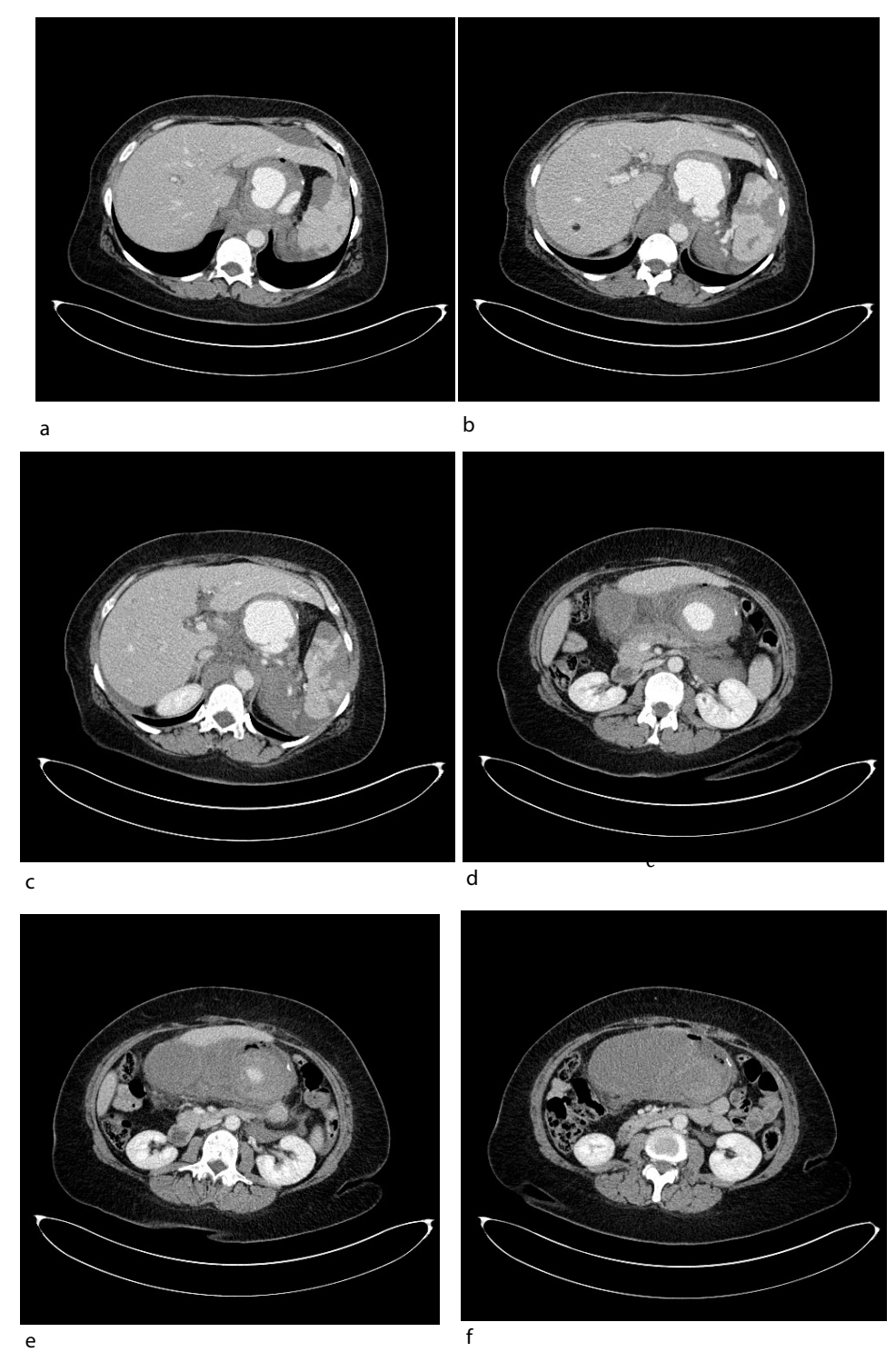

Figure 1. CT axial images after intravenous injection of iodinated contrast media show a, b) a well defined mass with strong enhancement after contrast medium injection that suggests the presence of a large aneurysm of splenic artery in communication with gastric wall; c, d) the vascular lesion is not separated from the gastric wall and surgical clips of previous Sleeve gastrectomy are partially seen; e, f) distended and thick-walled gastric tubule due to hematoma of the lateral wall is seen. Note also the stranding of the retroperitonuem, especially around the pancreas

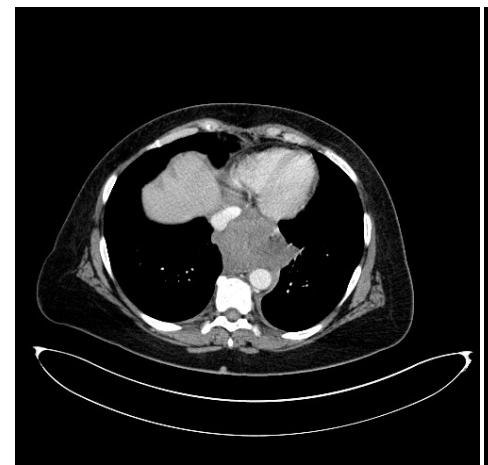

a

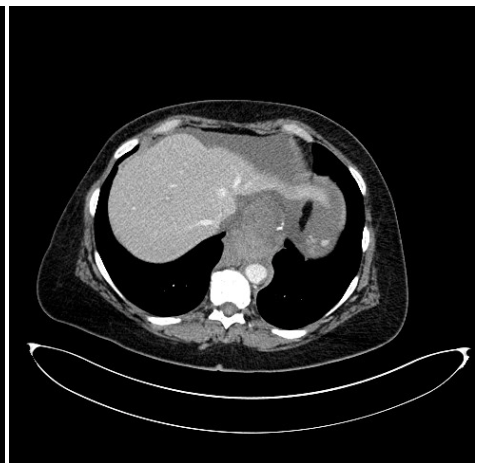

b

Figure 2. CT axial images after intravenous injection of iodinated contrast agent demonstrate a, b) the presence of mediastinal cloth, with distal intrathoracic esophagus compression 


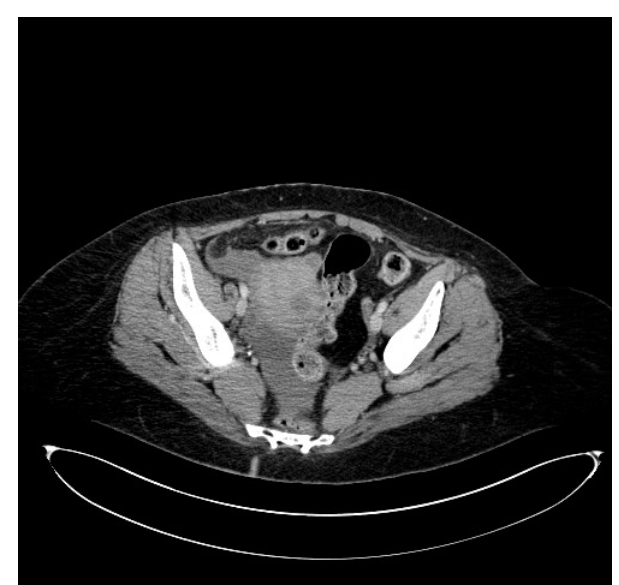

Figure 3. CT axial image after intravenous injection of iodinated contrast agent at the pelvis shows the presence of fluid collection due to hemoperitoneum

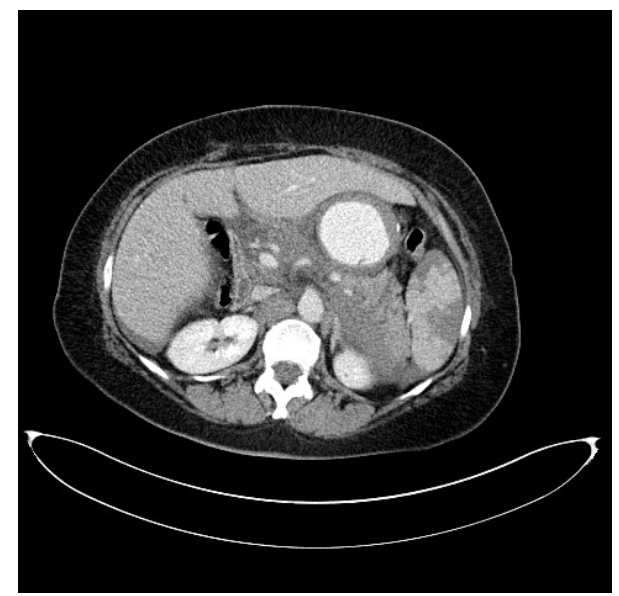

Figure 4. CT axial image after intravenous injection of iodinated contrast medium: evidence of hypodense triangular areas from the splenic hilum to the periphery, of the spleen due to ischemic injury

arteritis. They are extremely fragile and often cause intermittent and repetitive bleeding rather than massive haemorrhage $[8,9]$.

It should be noted that a useful distinction has been made between true aneurysms and pseudoaneurysms based on some characteristics.

True aneurysms of the splenic artery are usually smaller than $3 \mathrm{~cm}$, with possibility to range until $9 \mathrm{~cm}$. They may be multiple and are most commonly located in the distal portion of the artery, commonly with peripheral calcification in atherosclerotic contest $[10,11]$.

Clinically, most SAAs are asymptomatic [11] and the rupture rates reported is closer to $2-3 \%$ [12].

Typical clinical presentations of ruptured SAA include abdominal pain, hemodynamic instability, and gastrointestinal bleeding [13].

On the other hand, the mean size of splenic artery pseudoaneuryms was $4.8 \mathrm{~cm}$ (range $0.3-17 \mathrm{~cm}$ ) and the etiology includes pancreatitis, trauma, iatrogenic and previous surgery, and, rarely, peptic ulcer disease $[1,12]$.

In the case of pancreatitis, pancreatic enzymes are thought to cause a necrotizing arteritis with destruction of vessel wall architecture and fragmentation of elastic tissues, leading to aneurysm or pseudoaneurysm [14].
Unlike SAAs, splenic artery pseudoaneurysms nearly always present with symptoms. In a large review of the literature, only $2.5 \%$ of cases presented incidentally. The most common presentations are abdominal pain (29.5\%), hematochezia or melena (26.2\%), hemorrhage into the pancreatic duct (20.3\%), and hematemesis $(14.8 \%)[1,12]$.

Secondary hemorrhage from pseudoaneurysms may involve the pancreatic duct, peritoneum, retroperitoneum, adjacent organs as stomach and colon, or a pseudocyst if one is present $[1,12,14,15]$.

The risk of rupture of a splenic artery pseudoaneurysm can be as high as $37 \%$, with the mortality rate approaching $90 \%$ when untreated [16].

The CT appearance of SAA and pseudoaneurysm can be difficult to differentiate and the clinical history can often help to distinguish true SAA from pseudoaneurysms [12].

A true SAA usually appears as a well-defined and homogeneous contrast-enhanced mass; peripheral calcifications or mural thrombus may also be visible [13].

In case of pseudoaneurysm of the splenic artery a hyperdense round lesion is typically visible with a reported mean value of $40 \mathrm{HU}$ [17], showing focal strong enhancement after administration of contrast material, similar to that of blood vessels [12]. This focal region of strong enhancement may be surrounded by a thrombus or hematoma [13].

Three-dimensional rendering improves evaluation of the splenic artery, readily distinguishing tortuous vessel from aneurysm [18].

The choice of operation, however, is determined by location of the vascular lesion and by risk of rupture. Treatment should be always undertaken in case of increased risk of rupture, growth and symptomatic patients [19].

In conclusion, iatrogenic causes of splenic artery pseudoaneurism are very rare. In a review of 157 cases over 35 years, the authors found four cases with presumed iatrogenic causes [13].

One of them presented as an intraperitoneal rupture 14 years after splenectomy for trauma that was complicated at that time by a subphrenic abscess [20]. Other iatrogenic causes were Rouxen-Y pancreaticojejunostomy [21] and placement of percutaneous transgastric catheter for a pancreatic pseudo- cyst [22].

Upadhyaya et al. [7] reported a similar case of adventitia of the distal splenic artery probably damaged by the prolene stitch taken during placement of the reinforcing layer to the gastric sleeve anastomosis. Alternatively, they proposed the hypoteshis that the prolene suture could gradually have eroded into the splenic arterial wall to form a splenic artery pseudoaneurism.

In our patient, CT examination revealed that splenic artery aneurysm wall was in close proximity to surgical staple line of previous Sleeve gastrectomy and a relation between them was present, as detected by CT examination and surgical exploration.

However, the splenic artery is in close proximity to the stomach and is likely to be injured during stomach mobilization.

\section{Learning points}

- As illustrated by this case, the possibility of pseudoaneurysm rupture should be kept in mind in cases of gastrointestinal bleeding in patients with previous surgery in the peripancreatic area, especially to lower the high-mortality rate associated with undiagnosed lesions. 
- CT examination is a valuable imaging technique for the diagnosis of pseudoaneurysmatic change of splenic artery.

- Finally, because the natural history of asymptomatic splenic artery pseudoaneurysm is unknown, Radiologists contribute should help in deciding between the conservative and surgical or endovascular treatment

\section{Informed consent}

Written informed consent for the case to be published (including accompanying images, case history, and data) was obtained from the patient.

\section{Declaration of interest}

The authors declare that they have no competing interests.

\section{References}

1. Tessier DJ, Stone WM, Fowl RJ, Abbas MA, Andrews JC, et al. (2003) Clinical features and management of splenic artery pseudoaneurysm: case series and cumulative review of literature. J Vasc Surg 38: 969-74. [Crossref]

2. Agrawal GA, Johnson PT, Fishman EK (2007) Splenic artery aneurysms and pseudoaneurysms: clinical distinctions and CT appearances. AJR Am J Roentgenol 188: 992-9. [Crossref]

3. Goldberg RF, Maley W, Kennedy EP, Yeo CJ, Lavu H (2011) Giant splenic artery pseudoaneurysm. J Gastrointest Surg 15: 1063-6. [Crossref]

4. Al-Habbal Y, Christophi C, Muralidharan V (2010) Aneurysms of the splenic artery - a review. Surgeon 8: 223-231. [Crossref]

5. Stanley JC, Wakefield TW, Graham LM, Whitehouse WM Jr, Zelenock GB, et al (1986) Clinical importance and management of splanchnic artery aneurysms. $J$ Vasc Surg 3: 836-40. [Crossref]

6. Trastek VF, Pairolero PC, Joyce JW, Hollier LH, Bernatz PE (1982) Splenic artery aneurysms. Surgery 91: 694-699. [Crossref]

7. Upadhyaya PK, Chava S, Bin-Sangheer S, Sudan R, Mittal SK, et al. (2008) Delayed rupture of a splenic artery pseudoaneurysm after biliopancreatic diversion. Obes Surg 18: 890-2. [Crossref]

8. Woods MS, Traverso LW, Kozarek RA, Brandabur J, Hauptmann E (1995) Successful treatment of bleeding pseudoaneurysms of chronic pancreatitis. Pancreas 10: 22-30. [Crossref]
9. El Hamel A, Parc R, Adda G, Bouteloup PY, Huguet C, et al. (1991) Bleeding pseudocysts and pseudoaneurysms in chronic pancreatitis. Br J Surg 78: 1059-1063. [Crossref]

10. Dave SP, Reis ED, Hossain A, Taub PJ, Kerstein MD, et al. (2000) Splenic artery aneurysm in the 1990s. Ann Vasc Surg 14: 223-229. [Crossref]

11. Abbas MA, Stone WM, Fowl RJ, Gloviczki P, Oldenburg WA, et al. (2002) Splenic artery aneurysms: two decades experience at Mayo clinic. Ann Vasc Surg 16: 442-449. [Crossref]

12. Saba L, Anzidei M, Lucatelli P, Mallarini G (2011) The multidetector computed tomography angiography (MDCTA) in the diagnosis of splenic artery aneurysm and pseudoaneurism. Acta Radiol 52: 488-498. [Crossref]

13. Agrawal GA, Johnson PT, Fishman EK (2007) Splenic artery aneurysms and pseudoaneurysms: clinical distinctions and CT appearances. AJR Am J Roentgenol 188: 992-9. [Crossref]

14. Puri S, Nicholson AA, Breen DJ (2003) Percutaneous thrombin injection for the treatment of a post-pancreatitis pseudoaneurysm. Eur Radiol 13 suppl 4: L79-L82. [Crossref]

15. Flati G, Andren-Sandberg A, La Pinta M, Porowska B, Carboni M (2003) Potentially fatal bleeding in acute pancreatitis: pathophysiology, prevention and treatment. Pancreas 26: 8-14. [Crossref]

16. Huang IH, Zuckerman DA, Matthews JB (2004) Occlusion of a giant splenic artery pseudoaneurysm with percutaneous thrombin-collagen injection. J Vasc Surg 40: 574 577. [Crossref]

17. Yamaguchi K, Futagawa S, Ochi M, Sakamoto I, Hayashi K (2000) Pancreatic pseudoaneurysm converted from pseudocyst: transcatheter emboliza-tion and serial CT assessment. Radiat Med 18: 147-50. [Crossref]

18. Itoh K, Kamiya Y, Ohno N, Togawa S, Nomura T, et al. (2002) A case of pancreaticoduodenal artery aneurysm causing pancreatic pseudotumor and duodenal obstruction. Eur J Gastroenterol Hepatol 14: 457-461. [Crossref]

19. Maharaj R, Raghunanan B, Mohammed W, Rambally R, Sookdeo VD, et al. (2018) A rare case of massive lower gastrointestinal bleeding from a ruptured splenic artery aneurysm. J Surg Case Rep 2: 1-4. [Crossref]

20. Toscano RL, Ruiz OR, Gerace CA Jr (1995) Rupture of splenic artery pseudoaneurysm. Am Surg 61: 940-942. [Crossref]

21. Smith JA, Macleish DG, Collier NA (1989) Aneurysms of the visceral arteries. Aust $N$ Z J Surg 59: 329-334. [Crossref]

22. Quinn SF, Finney R, Rosemurgy A, Pieck CG (1988) Splenic artery pseudoaneurysm after placement of percutaneous transgastric catheter for a pancreatic pseudocyst. AJR Am J Roentgenol 151: 495-6. [Crossref]

Copyright: (C2019 Ricci D. This is an open-access article distributed under the terms of the Creative Commons Attribution License, which permits unrestricted use, distribution, and reproduction in any medium, provided the original author and source are credited. 